\title{
Connecting Recurrent Novae to [Some] Type Ia Supernovae
}

\author{
Ferdinando Patat \\ European Southern Observatory, \\ K. Schwarzschild-Str. 2, D-85748, Garching b. München, Germany \\ email: fpatat@eso.org
}

\begin{abstract}
In this review, I summarize the observational attempts made so far to unveil the nature of the progenitor system(s) of Type Ia supernovae. In particular, I focus on the most recent developments that followed the claimed detection of circumstellar material around a few events, and on the link this possibly establishes with recurrent novae. In this framework, I then discuss the case of RS Oph, what we know of its circumstellar environment, and what this is telling us about its supposed connection to Type Ia supernovae explosions.
\end{abstract}

Keywords. stars: novae, supernovae, binaries, individual (RS Oph)

Going after one beloved star today

and after another beloved star tomorrow

will not bring us very far.

Alvio Renzini (2011)

\section{Introduction}

There is no need here to spell out why it is important to understand the nature of Type Ia supernovae (SNe Ia) progenitors. During this conference this has been clearly and explicitly stated on many occasions.

Starting during the early seventies, when the currently accepted general binary scenario was proposed (Whelan \& Iben 1973), the question mark about the nature of the donor and the accreting star has been growing bigger and bigger. In recent years the seminal question "single- or double-degenerate?" has evolved, and we are now asking ourselves what fraction each of the two channels (hereafter SD and DD, respectively) contribute to the global rate of SNe Ia. In the case of the $\mathrm{SD}$, we would also like to know what is the contribution of theme's variations, where the donor star is a red giant, a subgiant, a main-sequence star, or a He-star. The problem has become a challenge that many groups, both theoretical and observational, have taken.

In this review I will focus on the observational side, going through the various attempts that have been made so far and the recent developments. Here I am on purpose neglecting the SNe Ia rate approach (see for instance Greggio 2010 and references therein), as this is treated in a number of contributions in these proceedings.

\section{In Quest of the Prey}

A number of different ways of gaining insights to the progenitor's nature were explored. These are recapped in the following sub-sections, along with their pros and cons. 


\subsection{Direct Observation of the Progenitor System}

This obviously provides the most direct information on the system. So far three different tracks have been beaten:

(a) If the system is formed by a WD accreting material from a non-degenerate companion star, the latter is supposed to survive the explosion. As a consequence of the WD disruption, it gets a gravitational kick, and it runs away at an unusually high speed (when compared to all other field stars). On the contrary, if the SN is the outcome of the merger of 2 WDs, then there should be no high-speed star left over. Since this requires multi-epoch accurate astrometry of the field surrounding the SN site, the method can only be applied to historical SNe in the Galaxy. In particular, Ruiz-Lapuente et al. (2004) have identified what might be the donor star to Tycho SN exploded in 1572. This would clearly testify in favor of a single-degenerate system for this specific event. However, the finding has been questioned by Kerzendorf et al. (2009, 2011, see also these proceedings), who did not identify an unambiguous donor star candidate.

(b) SD progenitor systems are supposed to spend some million years in a phase of steady nuclear burning while accreting matter from the donor. In virtue of this fact, they should be detectable as super-soft X-ray sources (SSS) (Di Stefano 2010a). Under certain circumstances also DD systems may undergo a SSS phase, but with X-ray luminosities one order of magnitude lower than in the previous case (Yoon, Podsiadlowski \& Rosswog 2007, Di Stefano 2010b). Therefore, the detection of SSS coincident with a SN site would allow a direct study of [some of] the progenitor properties. This methods has been first applied to SN 2007on, for which pre-explosion X-ray archival data were available (Voss \& Nelemans 2008). The claim of a detection (and the consequent conclusions on the progenitor's nature) was questioned by Roelofs et al. (2008). A similar study was conducted on the recent, nearby SN 2011fe. However, only upper limits on the X-ray luminosity could be set, and they are not sufficiently deep to disentangle between the two scenarios (Li et al. 2011). The main limit of this method is that it requires pre-explosion archival X-ray images of the SN site. This problem might be mitigated by a deep X-ray survey of a sample of nearby galaxies.

(c) In absolute terms, prior to the explosion the progenitor WD is a faint star. Therefore, its direct optical detection is out of discussion, at least for extra-galactic events. However, the companion star might be detectable, depending on its luminosity and this opens a possibility similar to the analog for core-collapse events (Smartt 2009). This was attempted for a number of SNe Ia (see Maoz \& Mannucci 2008, Smartt 2009), but the limits were not sufficiently stringent to rule out any of the possibilities. Things changed substantially with the very recent discovery of the nearby SN $2011 \mathrm{fe}$ in M101 $(d \approx 6$ Mpc). Based on pre-explosion HST images Li et al. (2011) were able to place a limit that rules out a red-giant star, and hence a symbiotic system like RS Oph, for this particular event. The He-star channel is only partially excluded, while a system with a MS-star transferring mass via Roche lobe overflow is fully consistent with the data, as is a DD configuration.

The methods listed so far provide direct properties of the progenitor. However, at least with the current observing capabilities, they can only be applied to a small number of objects, which makes them not really suitable for a statistical study. In the following, I discuss what can be referred to as indirect methods. They have the advantage over the direct methods of being applicable to a larger number of objects. However, they suffer from the fact that the information they provide is not directly related to the progenitor, but to what can be deduced from its surroundings. 


\subsection{Search for Entrained Material}

The impact of the SN ejecta on the companion star is expected to strip its envelope (in the case this is not a WD). Part of it becomes entrained in the ejected material and should be observable at late phases in the form of narrow emission lines (Wheeler et al. 1975, Fryxell \& Arnett 1981, Taam \& Fryxell 1984, Chugai 1986 Livne et al. 1992, Marietta et al. 2000, Meng et al. 2007, Pakmor et al. 2008). So far, no trace of hydrogen (or helium) has been detected in the late spectra of Type Ia SNe and this rules out systems with secondary stars close enough to the exploding WD to be experiencing Roche-lobe overflow at the time of explosion (Leonard 2007).

\subsection{High-Velocity Ca II Features in Early SNe Ia Spectra}

A feature which is common to most of SNe Ia is the presence of high-velocity components in the Ca II NIR triplet (Wang et al. 2003, Mazzali et al. 2005). Gerardy et al (2004) have explained these features as formed within a high velocity shell produced by the interaction between the SN ejecta and small amounts of circumstellar material $\left(M \leqslant 10^{-2} M_{\odot}\right)$. The models show that this material, which must be very close to the exploding star (i.e. within a few $10^{15} \mathrm{~cm}$ ), would not be revealed in any other form (variations of the light curve or appearance of narrow emission lines; see also next sub-section). The CSM material might come from the accretion disk, the Roche lobe, or the common envelope (Gerardy et al. 2004). It must be noticed that these features can be explained by a $3 \mathrm{D}$ structure of the explosion (Mazzali et al. 2005) and, therefore, circumstellar interaction is not necessarily a unique interpretation (Quimby et al. 2006).

\subsection{Search for Signs of Ejecta-CSM Interaction}

The previous method is supposed to probe the immediate surrounding of the SN. However, if the donor is a red giant, one expects to have a significant amount of matter at larger distances from the progenitor. The SN ejecta will sooner or later crash into it, and some fraction of the kinetic energy will be converted into optical, UV, and radio emission. This has been the most systematically pursued channel for addressing our question. However, all attempts to detect direct signatures of circumstellar material in normal SNe Ia using this method have failed, and only upper limits to the mass loss rate could be placed from optical (Lundqvist 2003, 2005; Mattila et al. 2005), radio (Panagia et al. 2006, Chomiuk et al. 2011), and UV/X-Ray emission (Immler et al. 2007).

Assuming a typical $\rho \propto r^{-2}$, spherically symmetric, steady red-giant wind with a velocity of $10 \mathrm{~km} \mathrm{~s}^{-1}$, the most stringent VLA non-detections imply mass loss rates below a few $10^{-8} \mathrm{M}_{\odot} \mathrm{yr}^{-1}$ (cf. the case of SN 2006X, Stockdale et al. 2006). Before the start of E-VLA operations, the lack of radio detection could exclude only the symbiotic channels with high mass loss rates $\left(10^{-5}-10^{-6} \mathrm{M}_{\odot} \mathrm{yr}^{-1}\right)$. However, the augmented sensitivity of E-VLA has now pushed down this limit quite significantly. The conclusion is that either all SNe Ia observed with the refurbished VLA facility come from DD systems, or the CSM scenario adopted so far to interpret the radio data needs to be radically reconsidered (Chomiuk et al. 2011).

\subsection{Study of SN Remnants}

The properties of young SN remnants are linked to the structure of the medium in which they expand. In turn this is related to the material outflows from the progenitor system before it exploded (both from the RG and from the accretion winds that are supposed to come from the WD). Therefore, some independent information on the progenitor can be gained looking at young SN remnants (Badenes et al. 2007; Reynolds et al. 2007; Chiotellis et al. 2011). Although the application of the method is still in its youth, and 
is significantly limited by the [so far] small number of remnants that can be studied, it will certainly provide additional constraints.

\section{CSM Detection via Absorption Lines}

All the indirect methods presented above require a direct interaction between the SN ejecta and the circumstellar material. But what about cavities around the SN? If novalike evacuation mechanisms like those proposed by Wood-Vasey \& Sokoloski (2006) are at work, then the immediate surroundings of the explosion site may exhibit a complex geometry because of the presence of an accretion disk, the bi-polar structure of the recurrent nova $(\mathrm{RN})$ ejecta, and possible fragmentation of the ejecta. Because of this, and especially during the early epochs of SN evolution, it is possible that we do not see signs of interaction (down to the E-VLA limits) just because there is no (or not enough) circumstellar material. In addition, if the material is confined within a series of nested (and fragmented) shells, then the interaction may last only a limited amount of time, hence making its detection via radio and/or X-ray emission more difficult.

With these speculative considerations in mind, back in 2006 my collaborators and I started considering an alternative approach to the problem, which would allow the detection of small amounts of material without requiring direct interaction with the SN ejecta. This technique and its first application is presented in Patat et al. (2007), and further expanded in Simon et al. (2009) to which I refer the reader for a more quantitative treatment. The idea is based on the fact that, at variance with core-collapse SNe, Type Ia SNe are relatively weak UV sources because of strong line-blocking by heavy elements (Fe/Co/Ti/Cr; Pauldrach et al. 1996; Mazzali et al. 2000). As a consequence, their ability to ionize possible circumstellar matter at large distances is rather limited. Therefore, provided that the gas is placed along the line of sight, it can be revealed by optical absorption lines like Ca II H\&K, Na I D, and K I. These are all strong lines, so intense that they enable the detection of tiny amounts of material. As opposed to the emission lines that are expected to arise only in the case of direct ejecta-CSM interaction (mainly $\mathrm{H}$ and $\mathrm{He}$ ), absorption lines are generated by material placed virtually at any distance along the line of sight (and only along the line of sight, which is one of the drawbacks of the method).

Just because of this (one may object), there is in principle no difference between a line arising in the CSM and one generated within an ISM cloud placed along the line of sight. However, given its weak ionizing radiation field, an SN Ia is hardly capable of ionizing gas placed at distances larger than $\sim 10^{18} \mathrm{~cm}$. And even if it were, the typical ISM densities would imply very long recombination times, meaning that no time variations are expected in lines arising in the ISM. On the contrary, this may not be true for absorptions produced by dense gas close to the explosion site. If the density is high enough, the material would be initially (and possibly partially) ionized by the SN radiation field. Then, as the SN fades away, the material recombines, and the lines get more and more intense as time goes by. Obviously, at some point this material may also be reached by the SN ejecta. Although its mass could be insufficient to generate a measurable emission (and would therefore make any X-ray and radio detection fail), the interaction would re-ionize the material, causing the absorption features to disappear. Given that the SN ejecta velocity is known, one can deduce the distance of the material from the SN just from the timing of the line disappearance. The SN ejecta move at typical velocities of $3 \times 10^{4} \mathrm{~km} \mathrm{~s}^{-1}$. As an SN Ia rises to maximum light in about 20 days, this implies that at this epoch the ejecta have traveled $\approx 6 \times 10^{15} \mathrm{~cm}(\sim 400 \mathrm{AU})$. This gives an idea of the spatial scales that can be probed by this method. 
These ideas were first applied to the bright SN 2006X, for which we obtained a series of high resolution spectra with the VLT+UVES. When we compared the first two epochs (separated by two weeks around maximum light) we found the line profile of Na I D had profoundly changed. On the blue side of a deep absorption (which was easily identified as the signature of a thick molecular cloud associated with the local spiral arm) a series of weak features had appeared, indicating the presence of material moving towards the observer at a velocity between 50 and $100 \mathrm{~km} \mathrm{~s}^{-1}$ (Patat et al. 2007; Patat 2009). The conclusion was that these features were originating either in the wind of a red giant (RG), or in the remnant shells of successive nova outbursts. Both possibilities favored the SD scenario (but see footnote 1 in Patat 2009).

A second variability detection was reported for SN 2007le by Simon et al. (2009), who reached similar conclusions about the circumstellar nature of the material generating the variable $\mathrm{Na}$ I D absorption. As in the case of SN 2006X, no time evolution was seen in the Ca II H\&K profile, strongly testifying in favor of a radiation effect (as opposed to a geometrical effect; see Chugai 2008 and Patat et al. 2010). As of today, thirteen SNe Ia have been studied by means of multi-epoch, high-resolution spectroscopy (Cox et al. 2011, Patat et al. 2011b, Simon et al. 2010). Of these, only two have shown line variability†. This can be interpreted in terms of viewing angle effects, the presence of other SD sub-channels, or even DD. Although the observations are still in progress while this review is being written, I can anticipate that the high-resolution data obtained so far for the nearby SN 2011fe do not show any trace of Na I D variation. It is worth noting that for this one object we have the most stringent limit on the mass loss rate: prompt radio and X-Ray observations have yielded $d M / d t \leqslant 10^{-8}\left(\mathrm{w} / 100 \mathrm{~km} \mathrm{~s}^{-1}\right) \mathrm{M}_{\odot} \mathrm{yr}^{-1}$ (Horesh et al. 2011). Moreover, we know the progenitor is not in a symbiotic system that would undergo a RN phase (Li et al. 2011), so that no complex CSM structure is expected. Of course, SN 2011fe is just one object and it does not tell us how frequent these systems are. For instance, one might ask the question whether the frequency of events displaying $\mathrm{Na}$ I variations is related to the occurrence of a particular progenitor channel (more specifically the symbiotic channel). Although we are still far from being able to answer this question, some statistical evidence is emerging. The recent study by Sternberg et al. (2011) has shown a significant excess of blue-shifted $\mathrm{Na}$ I features in SNe Ia, which are interpreted as a systematic sign of gas outflows at velocities ranging from a few $10 \mathrm{~km} \mathrm{~s}^{-1}$ to more than $100 \mathrm{~km} \mathrm{~s}^{-1}$. These indicate that the SD channel (with possible sub-channels) dominates over the DD channel.

One issue with the conclusions drawn from the line variability studies is that we are not yet sure RNe do indeed generate structures in the CSM that i) have the required velocities, and ii) survive long enough to reach the required distances $\left(>10^{16} \mathrm{~cm}\right)$. At this point one needs to close the loop, asking oneself the question: do RN systems show similar features?

\section{Closing the Loop: the Case of RS Oph}

RS Oph contains a hot component that accretes material from a RG. It undergoes RN outbursts with a period of roughly 20 years, the last of which took place in February 2006 (see the reviews by Mikolajewska and Anupama in these proceedings). The hot component is identified as a WD close to the Chandrasekhar mass. For this reason,

$\dagger$ Another two cases of line variability were reported for SN 1999cl (Blondin et al. 2009) and SN 2006dd (Stritzinger et al. 2010). However, they are both based on low-resolution data, and not much can be said about the physical conditions of the gas in which the lines originate. 


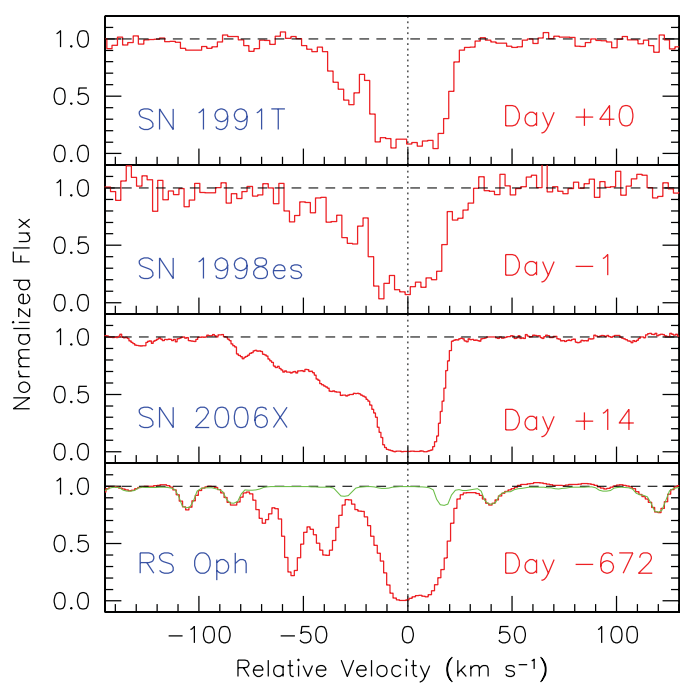

Figure 1. Comparison among high-resolution spectroscopy of three SNe Ia and RS Oph in the region of $\mathrm{Na} \mathrm{I} \mathrm{D}_{2}$. The velocity scales refer to the center of the deep absorption, most likely associated with the local spiral arm. The light colored curve in the bottom panel is an atmospheric model.

RS Oph has been proposed as a viable candidate to explode as an SN Ia, and it therefore constitutes a most suitable laboratory to address the above question. RNe offer the possibility of being studied before, during, and after the outburst, and this is a great advantage over SNe. We studied multi-epoch, high-resolution data taken across the 2006 outburst. The analysis is rather complex and the details can be found in Patat et al. (2011a). The main conclusion is that a number of blue-shifted Ca II, Na I, and K I absorption lines, with velocities reaching $50 \mathrm{~km} \mathrm{~s}^{-1}$, are associated with material not belonging to the circumbinary environment. The resemblance to what is seen in some SNe Ia is striking (Figure 1). These features, which during the outburst tend to weaken (or even disappear), show up again in the data taken about two years after the outburst, demonstrating that the gas in which they originate has survived the eruption, and must therefore be placed at distances larger than $\approx 3 \times 10^{15} \mathrm{~cm}$. The most natural explanation for these features is that they are generated in structures produced by the interaction between the previous nova shells and the RG wind lost in the inter-outburst phase. The relatively low velocities observed for these features require that the nova shell is significantly decelerated while expanding in the material lost by the RG. Is this physically possible within the framework of known facts about RN systems like RS Oph?

Assuming spherical symmetry and momentum conservation, and using the known parameters of the system (ejected mass, shell kinetic energy and initial velocity, interoutburst period, RG wind velocity), one can see that in order to decelerate the nova shell a mass loss rate of $3 \times 10^{-7} \mathrm{M}_{\odot} \mathrm{yr}^{-1}$ is required (Patat et al. 2011a). Despite the crude simplifications, this value is fairly consistent with what is expected for a RG in a symbiotic system: during the inter-outburst phase the RG loses enough material to slow down the next nova shell to velocities below $100 \mathrm{~km} \mathrm{~s}^{-1}$. Therefore, subsequent eruptions produce a series of nested structures, which would not physically interact with each other, but would rather remain separated in radius by $\sim 10^{15} \mathrm{~cm}$. Although this conclusion lends support to the proposed scenario, the simplified picture neglects two important facts: a) 
the nova ejecta deviate from spherical symmetry (O'Brien et al. 2006, Sokoloski et al. 2006, Ribeiro et al. 2009), and b) Rayleigh-Taylor instabilities lead to shell fragmentation. The hydrodynamical simulations presented during this conference by S. Mohamed (see these proceedings) have shown what kind of structures one is to expect and what the viewing angle effects would be. Indeed, the required structures, densities and filling factors seem to be consistent with the above picture. The remaining question is whether these structures would survive long enough to reach $10^{16}-10^{17} \mathrm{~cm}$, to make them viable for the SN case (SN ejecta are a factor of ten faster than in novae).

A number of questions remain unanswered. For instance, it is not yet clear whether the hot component in RS Oph is indeed a C-O or rather a He-Ne-Ng WD. This would of course make a big difference. Another relevant point to be addressed is whether what we have seen in RS Oph is common to all known RN systems. This will require a more systematic study of these systems, and may tell us whether the presence of narrow, blue-shifted absorption lines can be used to identify SNe Ia coming from this channel. Another fundamental piece of information we need is the survival time of the knots produced by the nova shell fragmentation. Future hydrodynamical simulations along the lines presented by Mohamed will have to extend over many nova outbursts to see whether these knots can reach the distances required to show up in SNe. Also, they will give us more quantitative estimates about the viewing angle effects, and what is to be expected when observing SN Ia.

Alvio Renzini is right, and the matter about SN Ia progenitors needs to be settled within the wider context of binary stellar evolution. But I believe that there is something to learn from single objects, like RS Oph, too (maybe just to reach the conclusion that such objects cannot explode as $\mathrm{SNe}$ Ia). It is probably the synergy between the two approaches that will lead us somewhere. All the pieces that at times (at least to me) seem just a contradictory set of information (as W. Hillebrandt once said half serious half joking, "everything taken into account, Type Ia SNe should never take place") may then fall together to form a comprehensive picture.

\section{References}

Badenes, C., Hughes, J. P., Bravo, E., \& Langer, N. 2007, ApJ, 662, 472

Blondin, S., et al., 2009, ApJ, 693, 207

Chiotellis, A., Schure, K. M., \& Vink, J. 2011, A\&SA submitted (arXiv:1103.5487)

Chomiuk, L., Soderberg, A. M., Chevalier, R., Badenes, C., \& Fransson, C. 2011, AAS, $21730405 \mathrm{C}$

Chugai, N. N. 1986, SvA, 30, 563

Chugai, N. N. 2008, Astronomy Letters, 34, 389

Cox, N., et al., 2011, in preparation

Di Stefano, R. 2010a, ApJ, 712, 728

Di Stefano, R. 2010b, ApJ, 719, 474

Fryxell, B. A. \& Arnett, W. D. 1981, ApJ, 243, 994

Gerardy, C. 2004, ApJ, 607, 391

Greggio, L. 2010, MNRAS, 406, 22

Horesh, A., et al., 2011, ApJ, submitted, (arXiv:1109.2912v1)

Kerzendorf, W., et al. 2009, ApJ, 701, 1665

Kerzendorf, W., et al. 2011, ApJ, in preparation

Immler, S. I., et al. 2006, ApJ, 648, L119

Leonard, D. C. 2007, ApJ, 670, 1275

Li, W., et al. 2001, Nature, submitted (arXiv:1109.1593) 
Livio, M. 2001, in Supernovae and gamma-ray bursts: the greatest explosions since the Big Bang, edited by M. Livio, N. Panagia and K. Sahu. STScI symposium series, Vol. 13. Cambridge, UK: Cambridge University Press, p. 334

Livne, E., Tuchman, Y., \& Wheeler, J. C. 1992, ApJ, 399, 665

Lundqvist, P., et al. 2003, in From twilight to highlight: the physics of Supernovae, ed. W. Hillebrandt \& B. Leibundgut (Berlin: Springer), 309

Lundqvist, P., et al. 2005, in Cosmic Explosions, ed. J. M. Marcaide, \& K. W. Weiler, CD-ROM version, IAU Coll., 192, 81

Maoz, D. \& Mannucci, F. 2008, MNRAS, 388, 421

Marietta, E., Burrows, A., \& Fryxell, B. 2000, ApJ, 128, 615

Mattila, S., et al. 2005, A\&\&A, 443, 649

Mazzali, P. A., et al. 2000, A\&A, 363, 705

Mazzali, P. A., Benetti, S., Stehle, M. et al. 2005, MNRAS, 357, 200

Meng, X, Chen, X. \& Han, Z. 2007, PASJ, 59, 835

OBrien, T. J., et al. 2006, Nature, 442, 279

Pakmor, R., Röpke, F. K., Weiss, A., \& Hillebrandt, W. 2008, A\& A, 489, 943

Panagia, N., et al. 2006, ApJ, 469, 396

Patat, F., et al. 2007, Science, 30, 490

Patat, F. 2009, in Probing stellar populations out to the distant universe, AIP Conference Proceedings, Volume 1111, p. 299

Patat, F., Cox, N. L. J., Parrent, J., \& Branch, D. 2010, A\&A, 514, 78

Patat, F., Chugai, N. N., Podsiadlowski, Ph., Mason, E., Melo, C., \& Pasquini, L., 2011a, A\&A, 530,63

Patat, F., et al. 2011, in preparation

Pauldrach, A. W., et al. 1996, A\&A A, 312, 525

Quimby, R., et al. 2006, ApJ, 636, 400

Renzini, A. 2011, public communication, this conference, closing remarks

Reynolds, S. P., et al. 2007, ApJ, 688, 135

Ribeiro, V. R. A. M., et al. 2009, ApJ, 703, 1955

Roelofs, G., Bassa, C. Voss, R., \& Nelemans, G. 2008, MNRAS, 391, 290

Ruiz-Lapuente, P., et al. 2004, Nature, 431, 1069

Simon, J., et al. 2009, ApJ, 702, 1157

Simon, J., et al. 2010, private communication

Smartt, S. J. 2009, ARAA, 47, 63

Smartt, S. J. 2011, these proceedings

Sokoloski, J. L., Luna, G. J.,M., Mukai, K., \& Kenyon, S. J. 2006, Nature, 442, 276

Sternberg, A., et al. 2011, Science, 333, 856

Stockdale, C. J., et al. 2006, CBET 396

Stritzinger, M., et al. 2010, AJ, 140, 2036

Taam R. E. \& Fryxell, B. A. 1984, ApJ, 279, 166

Voss, R. \& Nelemans, G. 2008, Nature, 451, 802

Wang, L., Baade, D., Höflich, P., et al. 2003, ApJ, 591, 1110

Whelan, J. \& Iben, I. 1973, ApJ, 186, 1007

Wheeler, J. C., Lecar, M., \& McKee, C. F. 1975, ApJ, 200, 145

Wood-Vasey, W. M. \& Sokoloski, J. L. 2006, ApJL, 645, 53

Yoon, S.-C., Podsiadlowski, P., \& Rosswog, S. 2007, MNRAS 380, 933 